\title{
POTENTIAL USAGE OF LIPOSOME-ENCAPSULATED PHOSPHOR FOR IN VIVO IMAGING OF TISSUE OXYGENATION
}

\author{
Leu-Wei Lo ${ }^{1}$, JA-An A. Ho ${ }^{2}$, Yen-Hsiung Chang ${ }^{3}$, Chia-Hua Chang ${ }^{1}$, \\ ChUNG-SHI YANG ${ }^{2,4}$ \\ ${ }^{1}$ Division of Medical Engineering Research, National Health Research Institutes, Taipei \\ ${ }^{2}$ Department of Applied Chemistry, National Chi-Nan University, Pauli \\ ${ }^{3}$ School of Medicine, National Taiwan University, Taipei \\ ${ }^{4}$ Department of Education and Research, Taichung Veterans General Hospital, Taichung \\ Taiwan
}

\begin{abstract}
Oxygen-dependent quenching of phosphorescence can provide a quantitative measurement with high temporal resolution of tissue oxygenation in vivo. It is a real-time optical means for prognosis of diseases where the oxygen concentration is essential. Phosphorescence quenching is a noninvasive methodology, otherwise no more than minimally invasive as the phosphor is necessarily introduced into vasculature prior to the measurement. Oxyphor R2, a dendritic phosphor with twolayer of glutamates, is a suitable phosphor for oxygen measurements owing to its high water solubility. We used a frequency-domain, phase modulation based instrument to calibrate Oxyphor $R 2$. The acquired quenching constant $\left(k_{Q}\right)$ and lifetime at zero oxygen $\left(\tau^{\circ}\right)$ were consistent with those calibrated from conventional time-domain instrument. Administered with Oxyphor R2, the rat hepatic oxygen distributions were imaged throughout the course of ischemia and reperfusion. After 5 min ischemia and subsequent 20 min reperfusion, distinct ischemic areas on the hepatic tissue were observed. In order to extend the application of in vivo oxygen imaging using phosphorescence quenching by minimizing the possible immunoresponse induced by phosphor; we are the first to co-synthesize the dipalmitoylphosphatidylglycerol (DPPG)-rich liposome with Oxyphor R2 to generate the liposome-encapsulated Oxyphor R2. Its calibration using the frequency domain measurement displayed higher quenching constant and shorter lifetime at zero oxygen $\left(k_{O}=1186\right.$ $\left.m m H^{-1} \mathrm{sec}^{-1}: \tau^{\circ}=150 \mu \mathrm{sec}\right)$ comparing to original Oxyphor $R 2\left(k_{O}=438 \mathrm{mmHg}^{-1} \mathrm{sec}^{-1}: \tau^{\circ}=630\right.$ usec). It implicated that the liposome-encapsulated Oxyphor R2 designed to neutralize the immunoresponse could be further applied to measure the tissue oxygenation in vivo, especially those with low oxygen concentration such as tumor:
\end{abstract}

Biomed Eng Appl Basis Comm, 2004 (August); 16: 224-232.

Keywords: liposome, dendritic porphyrin, phosphorescence quenching, ischemia and reperfusion, hepatic oxygenation.

Received: Feb 25, 2004; Accepted: July 25, 2004

Correspondence: Leu-Wei Lo, Ph.D., Assistant Investigator

Division of Medical Engineering Research, National Health Research Institutes, Taipei 114, Taiwan E-mail: lwlo@nhri.org.tw

\section{INTRODUCTION}

Functional tissue imaging has been recognized as a pivotal tool for clinical assessment due to its ability to provide both morphological and metabolic information that pertinent to the specific disease 
prognosis. Understanding that oxygen is an essential metabolite and whose disturbance is underlying to various pathophysiological states, a functional tissue imaging methodology based on oxygen-dependent quenching of phosphorescence has been continuously developed and validated as an effective method to noninvasively measure the oxygen concentration in biological systems [1-2]. It can provide rapid and accurate measurements of tissue oxygen levels or two dimensional imaging of oxygen map through thickness of tissue [3-4]. The recent development of this method has been advanced to non-invasively assess the in vivo oxygen distribution in tissuc by using a multifrequency system to perform the time-resolved phosphorescence analysis [5]. The capability of detcrmining the distribution of oxygen (oxygen histogram) in the microvasculature of tissue was further demonstrated with measurements of the oxygenation of subcutaneous Q7 tumors on Buffalo rat models [6].

Based on the principle of the phosphorescence quenching $[1,7]$, a standard measurement is proceeded with injection of phosphorescence compound into the blood vessel and followed by using a bifurcated light guide to conduct light from the excitation source to the surface of the tissue and to return the phosphorescence to the detector [5]. The oxygen concentration can be further determined by incorporating the measured phosphorescence lifetime into a well-defined relationship called the Stern-Volmer equation:

$$
\mathrm{PO}_{2}=\left(1 / \mathrm{k}_{\mathrm{Q}}\right)\left[\left(1 /(\tau)-\left(1 /\left(\tau^{\circ}\right)\right]\right.\right.
$$

, where $\tau^{0}$ is the lifetime in the absence of oxygen, $\tau$ is the lifetime at an oxygen pressure $\mathrm{PO}_{2}$, and $\mathrm{k}_{\mathrm{Q}}$ is a constant describing the quenching collisions between the phosphor molecules in the triplet state and molecular oxygen [8].

Generally there are two approaches for the instruments operating phosphorescence lifetime measurements. One is in frequency domain and the other is in time domain. The measurement in frequency domain is preferred to that in time domain for a variety of reasons including increase of signal to noise ratio [9]. Measurement of the fluorescence or phosphorescence lifetime in the frequency domain is generally called the harmonic or phase modulation method. The principle of using phase modulation method for the measurement of fluorescence or phosphorescence lifetime has been described in detail previously [10]. Nevertheless, for taking the advantage of current technology advances in fast gated, intensified CCD camera, the time domain data acquisition and analysis are prevailing in the implementation of fluorescence and phosphorescence lifetime imaging systems.
As a method for measuring oxygen, phosphorescence quenching has several desirable characteristics. First, it is a noninvasive optical method. When phosphors are dissolved in the blood, phosphorescence can be used for oxygen measurements in living tissue. Second, the response time is very short within milliseconds, and thus the measurements can be made in real time and are capable of following rapid transients in oxygen pressure. Third, if the phosphorescence quenching is well behaved, the dependence of phosphorescence lifetime on oxygen pressure follows a well-defined physical relationship, which can readily be expressed in a linear equation. This greatly facilitates data analysis. Fourth, in biological systems, phosphorescence quenching is highly specific for oxygen.

A fifth, and very valuable, characteristic of this method is that the calibration is dependent only on the phosphor and its molecular environment. When the environmental parameters are held constant, the calibration is absolute. This means that, unlike oxygen electrodes and most of the other available methods for measuring oxygen, once the calibration constants are determined they can be generally applied, independent of the operator or, within limits, the preparative procedures [9]. The calibration constants including the quenching constant and lifetime in the absence of oxygen need to be determined for physiological ranges of $\mathrm{pH}$, temperature and ionic strength. They can therefore be used by anyone wishing to make oxygen measurements under similar experimental conditions, including measurements of the oxygen pressure in blood in vivo. These values may also be used for verification of the performance of the phosphorescence measuring device and/or instrument settings [8].

For measuring oxygen concentration using phosphorescence quenching, one of the most readily available and easily used phosphors is Pd-meso-teta (4carboxyphenyl) porphyrin (PdTCPP). However, PdTCPP has to tightly bind to albumin prior to measurements in order to increase its water solubility [11]. To further improve the water solubility, a new class of porphyrins, based on dendritic construction of surround environment of the porphyrin core has been synthesized [12-13]. A two-layer of glutamates modified PdTCPP (dendrtic Oxyphor R2) provides high water solubility, no observed toxicity, good protection from chemically active components of tissue and appropriate size for excretion through kidney. In the present study, we calibrated Oxyphor R2 for oxygen measurement in frequency domain and validated all the quenching parameters consistent with those reported in previous study $[5,11]$. Furthermore, we demonstrated the applicability of Oxyphor R2 for in vivo measurement by acquiring the quantitative 
imaging of oxygen distribution in rat liver under ischemia and reperfusion with our implemented imaging system as stated in [8].

Except the necessary administration of phosphor molecules prior to the measurement, oxygendependent quenching of phosphorescence has been otherwise considered as a non-invasive optical tissue imaging method. Therefore, the increase of efficiency of in vivo oxygen measurement in terms of reducing immune response and increasing on-site delivery efficiency has to be emphasized. Biocompatible carrier, such as liposome, could be co-synthesized with phosphor molecules to generate liposome-encapsulated Pd-porphyrin to achieve these inquiries.

Liposomes have attracted considerable attention in recent years as model systems to study membrane phenomena, and also on account of potential applications as vehicles for drug delivery. For drug delivery applications, the liposomes can entrap soluble materials within the aqueous interior or alternatively hydrophobic materials can be bound to the bilayer membrane. By incorporating appropriate antibody while the formation of liposomes, it can render a target-search capability and provide the liposomes as on-site carriers for therapeutic agents. In addition, to avoid recognition by cell of the mononuclear phagocyte system (MPS) and uptake by the reticuloendothelial system (RES), several research groups have reported the increased localization of sterically stabilization liposome (PEG-liposomes) at tumor sites [14-16]. The long circulating "stealth" liposomes showed low toxicity and increased bioavailability.

In consideration of the phosphors used for oxygen measurements, it consists of two major components: optical chromophor and surrounding environment. Optical chromophor (OC) is a part of the phosphor, which is responsible for the phosphorescence. These useful properties are: (1) high absorption in the region of spectrum where natural chromophors of tissue, such as hemoglobin or myoglobin, do not absorb light, or absorb light only slightly; (2) phosphorescence with high quantum yields at room temperature $(>2 \%)$ and moderately long lifetimes $(0.1-1 \mathrm{msec})$ in the absence of oxygen. Surrounding environment (SE) is a ligand or set of ligands linked to a chromophor skeleton to provide an environment with the properties desirable for the oxygen measurements. Ideally, SE forms an inert globular structure around the chromophor which isolates it from alterations in the solvent, but through which small-uncharged molecules, such as oxygen, can penetrate to allow phosphorescence quenching. While encapsulating dendritic Pd-porphyrin such as Oxyphor $\mathrm{R} 2$, the unilamellar phospholipid may be considered as a modified SE in a larger scale and which may impose an impeding effect on oxygen diffusion towards to $\mathrm{OC}$.
In the present study, we are the first group to cosynthesize the dipalmitoylphosphatidylglycerol (DPPG)-rich liposome with Oxyphor R2 to generate the liposome-encapsulated Oxyphor R2 and for which, the de novo calibration of $\mathrm{k}_{\mathrm{Q}}$ and $\tau^{\circ}$ was preliminarily established as well to verify its further applicability for oxygen measurement in vivo.

\section{METHODS AND MATERIALS}

\subsection{Reagents and Materials for Liposome Preparation}

All inorganic chemicals and organic solvents used were reagent grade or better. Cholesterol, poly(vinylpyrrolidone) (PVP,MW 10 000), Tween-20, gelatin, Sephadex G-50, triethylamine(TEA), Trizma base (tris[hydroxymethyl]aminomethane, Tris), and $n$-octyl $\beta$-D-glucopyranoside (OG) were purchased from Sigma(St. Louis, MO). Dipalmitoylphosphatidylethanolamine (DPPE) and sulforhodamine B were purchased from Molecular Probes (Eugene, OR). Polycarbonate syringe filters of $3-, 0.4-$, and $0.2-\mu \mathrm{m}$ pore size were purchased from Poretics (Livermore, CA), and plastic-backed nitrocellulose membranes with pore size of $>3 \mu \mathrm{m}$ were from Schleicher and Schuell (Keene, NH). Dipalmitoylphosphatidylcholine (DPPC) and dipalmitoylphosphatidylglycerol (DPPG) were obtained from Avanti Polar Lipids (Alabaster, AL). $\mathrm{N}$ Succinimidyl-S-acetylthioacetate (SATA), 4(4-Nmaleimidophenyl)butyric acid hydrazide (MPBH), and $N$-ethylmaleimide (NEM)were purchased from Pierce (Rockford, IL). Carnation nonfat dry milk (NDM) powder was obtained locally.

Dye-loaded liposomes were prepared by the reversed-phase evaporation method [17]. The lipids mixture consisted of a 5:5:0.5:4 molar ratio of DPPC, cholesterol, DPPG, and DPPE-acetylthioacetate. The lipids were dissolved in $8 \mathrm{~mL}$ of a solvent mixture consisting of 6:6:1 volume ratios of chloroform, isopropyl ether, and methanol, followed by a 1-min sonication at $45^{\circ} \mathrm{C}$ under nitrogen. Then $1.4 \mathrm{~mL}$ of a warmed aqueous solution of $150 \mathrm{mM}$ Oxyphor R2 in TBS, adjusted to $\mathrm{pH} 7.0-7.5$ with $1 \mathrm{~N} \mathrm{NaOH}$, was added. After sonication of the solution for 6 min more, the organic solvent was removed by evaporating under nitrogen at $45^{\circ} \mathrm{C}$, leaving a dark purple, gel-like suspension of liposomes. An additional $2.6 \mathrm{~mL}$ of Oxyphor R2 dye was added, followed by another 5 min of sonication at $45^{\circ} \mathrm{C}$. Liposomes were kept warm before passing through three different pore sizes of prewarmed polycarbonate filters $(3.0,0.4$, and 0.2 $\mu \mathrm{m})$ to produce a homogeneous suspension. The liposomes were separated from unencapsulated 
Oxyphor R2 and traces of organic solvent by gel filtration on a $1.5 \times 25 \mathrm{~cm}$ Sephadex G-50 column at room temperature, followed by dialysis (MWCO, 12 $000-14000$ ) in $0.01 \mathrm{M}$ Tris- $\mathrm{HCl}, 0.15 \mathrm{M} \mathrm{NaCl}, \mathrm{pH} 7.0$ at $4^{\circ} \mathrm{C}$ in the dark.

\subsection{Stability Study and Characterization of Liposome}

Oxyphor R2 is a phosphorescence molecule; thus, the stability (intactness) of liposomes means the maintenance of their integrity, which can be determined by measuring phosphorescence intensity before and after lysis. Almost instantaneous lysis of liposomes was observed at room temperature when a solution of $n$-octyl $\beta$-D-glucopyranoside was added; total lysis of the liposomes was achieved by addition of $O G$ to a final concentration of $50 \mathrm{mM}$. For these phosphorescence tests, the Oxyphor R2 dye was excited at $525 \mathrm{~nm}$, and the phosphorescent emission intensity measurements were made at wavelength 596 $\mathrm{nm}$. Temperature effects on liposome stability were conducted by adding $10 \mu \mathrm{L}$ of liposome solution to 3 $\mathrm{mL}$ of osmotically balanced TBS, $\mathrm{pH} 7.0$, in a test tube preheated to the required temperature in a water bath. The liposomes were incubated for $5 \mathrm{~min}$ before measurement of phosphorescence intensity.

The diameter of the liposomes was measured with a Coulter LS particle size analyzer (Coulter Corp., Miami, FL) using the manufacturer's method. Liposomes were visualized by transmission electron microscopy of negatively stained preparations to check the integrity and size of the liposomes.

\subsection{In Vitro Calibration of Oxyphor R2 and Liposome-encapsulated Oxyphor R2}

As mentioned, the essence of the method of oxygen-dependent quenching of phosphorescence is the calibration, which depends only on the phosphor and its molecular environment. In order to precisely calibrate the quenching constants and lifetimes at zero oxygen, a special instrument and methodology were designed as described in [11]. In brief, Oxyphor R2 (Oxygen Enterprises, Philadelphia, PA) or liposomeencapsulated Oxyphor R2 was dissolved in a pH 7.4 buffer solution containing $20 \mathrm{mM}$ MOPS, $10 \mathrm{mM}$ $\mathrm{KH}_{2} \mathrm{PO}_{4}, 0.12 \mathrm{M} \mathrm{NaCL}$, and $2 \%$ by weight of bovine serum albumin to the final concentration of $10 \mu \mathrm{M}$. A round glass vial containing $2 \mathrm{ml}$ of phosphor solution to be calibrated was sealed with a stopper pad through which a polarographic oxygen electrode (World Precision Instrumentation) was inserted. The glass vial was thermostatted at $38^{\circ} \mathrm{C}$ and stirred with a magnetic stirring bar. Oxygen of the solution was slowly removed by passing a stream of purified argon gas. The phosphorescence signal was conducted through a bifurcated light guide that was arranged in a way for better representation of the lifetime near the tip area of the oxygen electrode. This can minimize the discrepancy caused by the oxygen gradient between the solution surface to the tip of the electrode. A frequency domain phosphorescence lifetime measuring system (PMOD 5000; Oxygen Enterprises, Philadelphia, PA) was used for the simultaneous measurement of the phosphorescence lifetime.

\subsection{Phosphorescence Imaging System for Oxygen Measurements}

The implementation of phosphorescence lifetime imaging system and the procedure to calculate the pixel by pixel oxygen concentration have been detailed described in our previous publication [8]. The specific delay time sequence selected for Oxyphor R2 was: 20, $40,80,160,300,480,760$, and $2500 \mu$ sec.

\subsection{Animal Preparation}

All animal procedures were in accordance with the National Health Research Institutes guidelines for the care and use of laboratory animals. Male SpragurDawley rats (260-350 gm) were deprived of food and had free access to water for 12 hours before the experiments. Rats were anesthetized with urethane (1.2 $\mathrm{g} / \mathrm{kg}$ body weight i.p.) and allowed to breathe spontaneously. The body temperature was maintained at $37^{\circ} \mathrm{C}$ with a heating plate. Polyethylene catheters were inserted into the femoral artery for intravenous administration (bolus) of Oxyphor R2 (50 mg/kg body $w t)$.

After a transverse upper abdominal laparotomy, th liver was mobilized and isolated by dividing all of its attachments with negligible bleeding. Temporal ischemia was introduced with the procedure similar to the portal triad clamping model (PTC model) that used total ligation of the vessels (hepatic artery, portal vain, and bile duct) in the hepatoduodenal ligament. The hepatic oxygen distribution was imaged after undergone $5 \mathrm{~min}$ ischemia and $20 \mathrm{~min}$ reperfusion.

\section{RESULTS}

\subsection{Characteristics of Oxyphor R2}

Oxyphor R2 was a Pd-meso-teta (4carboxyphenyl) porphyrin (PdTCPP)-dendrimer (fig. 1a). It was synthesized with two layers of glutamate modified on the core Pd-porphyrin, PdTCPP. The 16 carboxyl groups located on the outer layer of the dendritic Oxyphor $\mathrm{R} 2$ render total net 16 negative charges at near neutral $\mathrm{pH}$ which not only provide high water solubility but also prevent the molecule itself 
"leaking" from the blood vessels. These properties make Oxyphor R2 a prominent phosphor for measuring tissue oxygenation which represents the oxygen concentrations distributing in the tissue vascular system. The spectral analysis indicated that Oxyphor R2 has two absorption maxima at $421 \mathrm{~nm}$ (Soret-band) and $525 \mathrm{~nm}$ (Q-band), and a phosphorescence emission at $695 \mathrm{~nm}$ (fig. 1b). The amount needed for oxygen measurements is in the range of $15-60 \mathrm{mg} / \mathrm{kg}$ body wt.

\subsection{Hepatic Oxygen Distribution under Ischemia and Reperfusion}

The change of hepatic oxygen distribution under ischemia and reperfusion was monitored using phosphorescence imaging system. The imaged area of rat liver was first taken using reflected room light (fig. 2a) whose morphological features could be colocalized with later imaged oxygen maps. The hepatic oxygenation was heterogeneously distributed over the range of 30 to $55 \mathrm{mmHg}$ before the engagement of PTC ligation (fig. 2b). In contrast, after the course of 5 min ischemia with PTC ligation and subsequent 20 min reperfusion, the hepatic oxygen distribution showed the decrease of oxygen pressures to the range of 20 to $40 \mathrm{mmHg}$ and the distinct border zone of ischemic tissue (fig. 2c). All the oxygen maps were calculated from acquiring a series of intensity images with a specific delay time sequence selected for Oxyphor R2: $20 \mu \mathrm{sec}, 40 \mu \mathrm{sec}, 80 \mu \mathrm{sec}, 160 \mu \mathrm{sec}$, $300 \mu \mathrm{sec}, 480 \mu \mathrm{sec}, 760 \mu \mathrm{sec}$, and $2500 \mu \mathrm{sec}$. The ischemic area of the liver was contoured with prominent phosphorescence intensity after the 300 (sec intensifier gating delay, from which the oxygen pressure was calculated around $20 \mathrm{mmHg}$ (Data not shown). The image collected after a delay of 2500 (sec was used as the background and subtracted from each of the other phosphorescence images to prevent any light intensity arisen from non-phosphorescence sources into the integrated images.

\subsection{Characteristics of Liposome-encapsulated Oxyphor R2}

The characteristics of liposomes are listed in table 1. With liposomes of 346-nm diameter, it is possible to calculate that the average volume of a single liposome is $2.17 \times 10^{-11} \mu \mathrm{L}$ and the volume entrapped (assuming a bilayer thickness of $4 \mathrm{~nm}$ ) is $2.02 \times 10^{-11} \mu \mathrm{L}$. Assuming the dye concentration inside of the liposomes was equal to the original dye solution used, and comparing the fluorescence of lysed liposomes to that of standard Oxyphor R2 solutions, it is possible to calculate that there were $\sim 4.79 \times 10^{-10}$ liposomes $/ \mathrm{mL}$ and that each liposome contained $\sim 3.1 \times 10^{5}$ molecules of dye.
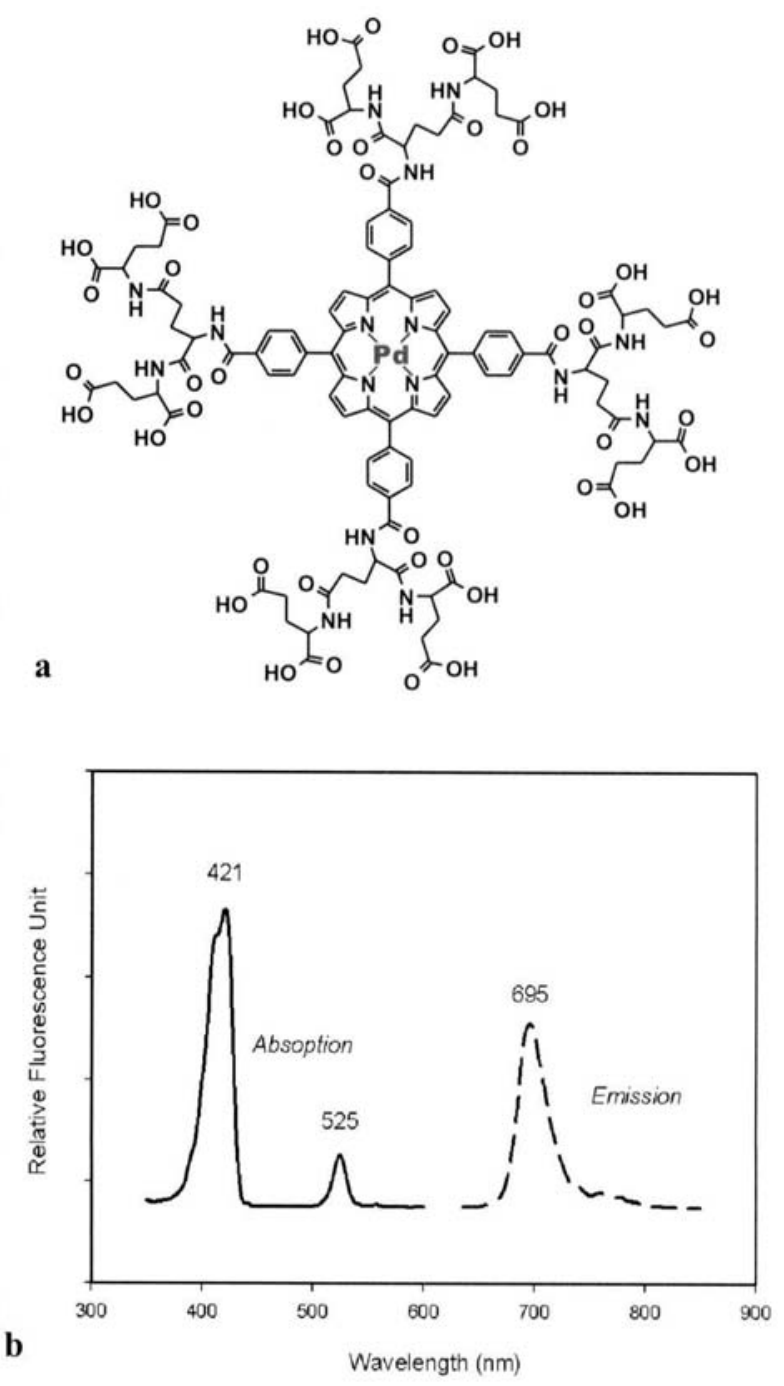

Fig 1. The chemical structure of Oxyphor R2, a Pdmeso-teta (4-carboxyphenyl) porphyrin (PdTCPP)dendrimer (a). It was synthesized with two layers of glutamate modified on the core Pd-porphyrin, PdTCPP. The spectral analysis indicated that Oxyphor R2 has two absorption maxima at $421 \mathrm{~nm}$ (Soret-band) and $525 \mathrm{~nm}$ (Q-band), and a phosphorescence emission at $695 \mathrm{~nm}$ (b).

In order to prevent the extravasation of liposomeencapsulated Oxyphor R2 from the vasculature, the liposomal lipid bilayer was carefully synthesized with a composition rich in dipalmitoylphosphatidylglycerol (DPPG), as described in materials and methods, so that to maintain the overall negative charge on the outer surface of liposome (fig. 3a). The spectral analysis demonstrated that the liposome-encapsulated Oxyphor R2 conserved the optical property of Oxyphor R2. It 


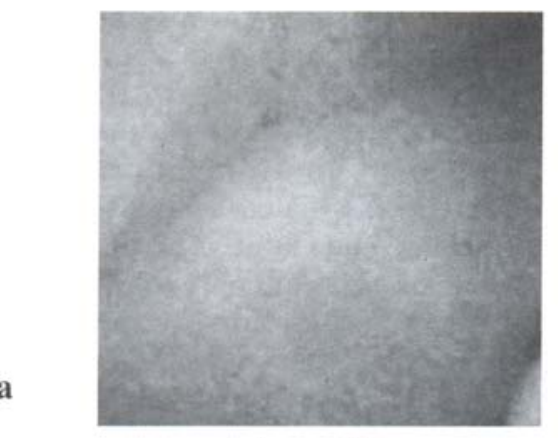

Liver Video
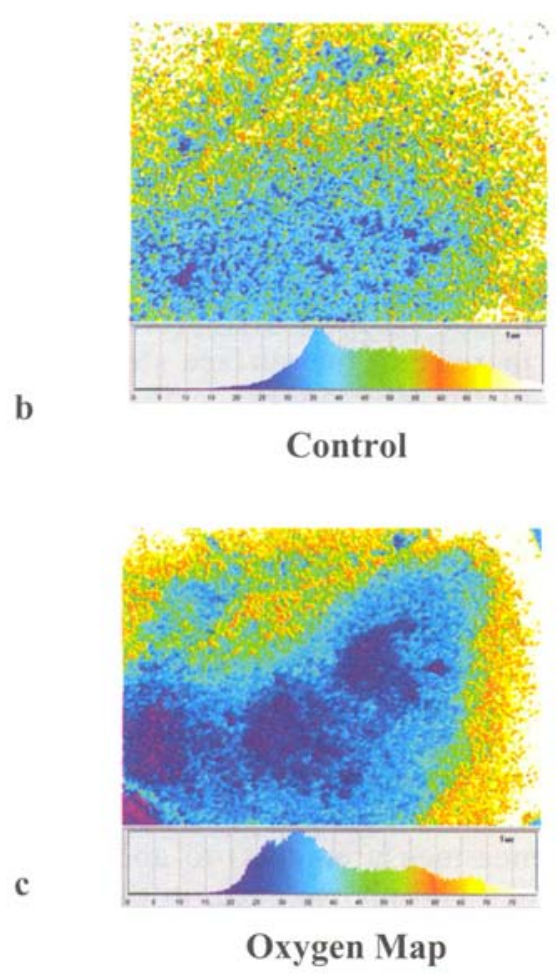

Fig 2. The change of hepatic oxygen distribution under ischemia and reperfusion was monitored using phosphorescence imaging system. (a) The imaged area of rat liver was first taken using reflected room light whose morphological features could be co-localized with later imaged oxygen maps. (b) The hepatic oxygenation was heterogeneously distributed over the range of 30 to $55 \mathrm{mmHg}$ before the engagement of PTC ligation. (c) After the course of $5 \mathrm{~min}$ ischemia with PTC ligation and subsequent $20 \mathrm{~min}$ reperfusion, the hepatic oxygen distribution showed the decrease of oxygen pressures to the range of 20 to $40 \mathrm{mmHg}$ and the distinct border zone of ischemic tissue.
Table 1. Characteristics of the Liposomes

\begin{tabular}{ll}
\hline mean diameter $(\mathrm{nm})$ & $346 \mathrm{~nm}$ \\
volume of liposome $(\mu \mathrm{L})$ & $2.17 \times 10^{-11}$ \\
entrap volume of liposome $(\mathrm{mL})$ & $2.02 \times 10^{-11}$ \\
liposome concentration $(\mathrm{number} / \mathrm{mL})$ & $4.79 \times 10^{10}$ \\
Oxyphor R2 concentration $(\mathrm{mM})$ & 26 \\
Oxyphor R2 molecules per liposome & $3.1 \times 10^{5}$ \\
\hline
\end{tabular}

has two absorption maxima at $418 \mathrm{~nm}$ (Soret-band) and $525 \mathrm{~nm}$ (Q-band), and a phosphorescence emission at $706 \mathrm{~nm}$ (fig. 3b). This observation further facilitates using liposome as a carrier for Oxyphor R2 for in vivo oxygen measurements as which would not disturb this dendrimer-porphyrin's optical characteristics.

\subsection{Calibrations of Oxyphor R2 and Liposome- encapsulated Oxyphor R2}

Phosphorescence quenching parameters for Oxyphor R2 with $2 \%$ bovine serum albumin with and without liposome encapsulation at $\mathrm{pH} 7.4,38^{\circ} \mathrm{C}$ were calibrated. The reciprocal of measured phosphorescence lifetimes versus corresponding oxygen pressure measured by polarographic oxygen clectrode was shown in figure 4 . According to SternVolmer equation, the slope of each plot represents its quenching constant $\mathrm{k}_{\mathrm{O}}$. The lifetime at zero oxygen $\tau^{\circ}$ could be acquired from the intercept of the plot. As indicated in figure 4 , the liposome-encapsulated Oxyphor R2 displayed higher quenching constant and shorter lifetime at zero oxygen $\left(\mathrm{k}_{\mathrm{Q}}=1186 \mathrm{mmHg}^{-1} \mathrm{sec}^{-1}\right.$; $\left.\tau^{2}=150 \mu \mathrm{sec}\right)$ comparing to Oxyphor $\mathrm{R} 2\left(\mathrm{k}_{\mathrm{Q}}=438\right.$ $\mathrm{mmHg}^{-1} \sec ^{-1} ; \tau^{\circ}=630 \mu \mathrm{sec}$ ).

\section{DISCUSSION}

We currently use phosphorescence probes that bind tightly to albumin. In in vitro experiments, albumin is added in molecular excess over the phosphor whereas in in vivo experiments there is an excess of albumin in the blood. The albumin-binding site provides a well-defined physical environment with a controlled access by oxygen. Since the environment of the phosphors independent of the medium (other than albumin), calibration of $\mathrm{k}_{\mathrm{Q}}$ and $\tau^{\circ}$ in vitro are fully valid for studies in vivo. In addition, as long as each is bound to albumin, in the concentration range of phosphors used in our studies there is no significant "self quenching" due to energy transfer from the triplet state to ground state phosphor molecules. To use liposome as Oxyphor R2 carrier, the liposomal lipid 


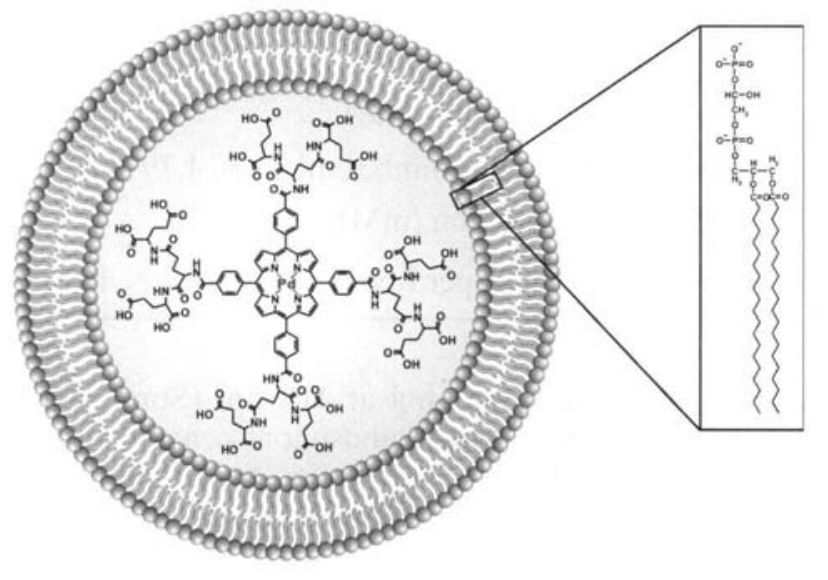

a

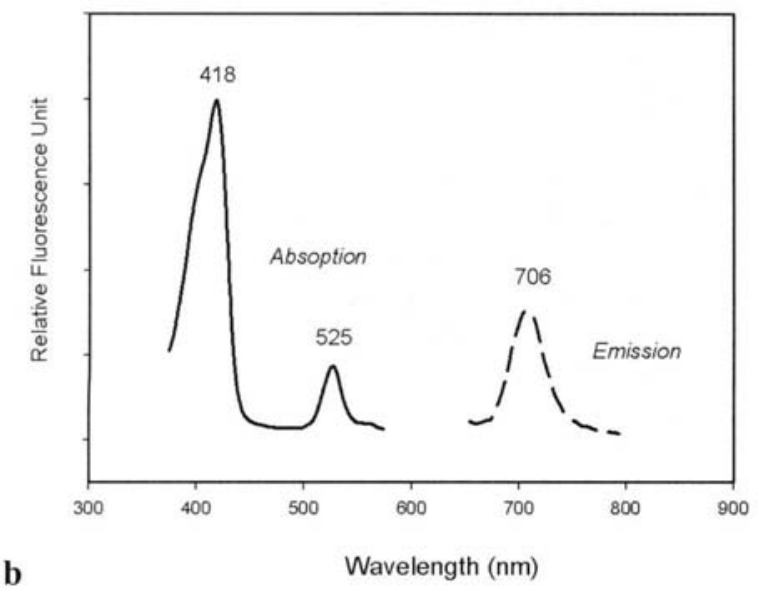

Fig 3. (a) Oxyphor $R 2$ was encapsulated with liposome, whose lipid bilayer was carefully synthesized with a composition rich in dipalmitoylphosphatidylglycerol (DPPG) so to maintain the overall negative charge on the outer surface. (b) The spectral analysis indicated that the liposome-encapsulated Oxyphor $R 2$ conserved the optical property of Oxyphor R2. It has two absorption maxima at $\mathbf{4 1 8} \mathrm{nm}$ (Soret-band) and $\mathbf{5 2 5}$ nm (Q-band), and a phosphorescence emission at $706 \mathrm{~nm}$.

bilayer was synthesized with a composition rich in dipalmitoylphosphatidylglycerol (DPPG) and thereby increasing the overall negative charge distribution on the surface of liposome to minimize the "leakage" of liposomes from blood vessels during their circulation. However, the bulk of negative charge would result in loose association of liposome-encapsulated Oxyphor $\mathrm{R} 2$ to albumin, which is composed of highly surface negative charges at $\mathrm{pH} 7.4$ as well. This may contribute at least in part towards disturbance of the well-defined physical environment provided by albumin and result in facilitated access by oxygen to incur phosphorescence quenching. The increase rate of collision between oxygen and Oxyphor R2 should engender the higher quenching constant $k Q$ of liposome-encapsulated Oxyphor R2 (1186 mm $\mathrm{mg}^{-1} \mathrm{sec}^{-1}$ to $438 \mathrm{mmHg}^{-1} \mathrm{sec}^{-1}$ ). Furthermore, the concentrated Oxyphor R2 confined in the liposomal internal compartment without being associated with albumin, the probability of self-quenching would significantly increase to which the much shorter $\tau$ was possibly attributed (150 $\mu \mathrm{sec}$ to $630 \mu \mathrm{sec})$. In order to increase the dynamic range of phoshorescence lifetime measurements, the high quenching constant $k_{Q}$ of liposome-encapsulated Oxyphor R2 would be reduced by modifying the liposomal surface with polyethylene glycol (PEG). With PEG conjugation, not only for the purpose of diminishing immune response [15-16], it could also impede oxygen diffusion in terms of dramatically increasing the surface steric effect of liposome, and thereby decrease the quenching constant.

However, while applying it to biological systems, the oxygen measurements have been limited to the surface layer of the tissue because the absorption bands of the Pd-meso-tetra-(4-carboxyphenyl)-porphyrindendrimer such as Oxyphor R2, were all at wavelengths less than about $540 \mathrm{~nm}$. At these wavelengths, absorption of the excitation light by chromophores in the tissue, notably hemoglobin, myoglobin, and cytochrome, limits the depths in tissue to which the excitation light can penetrate (and thereby phosphorescence measurements) to about $1 \mathrm{~mm}$ or less. The emitted phosphorescence, in contrast, has a maximum at $695 \mathrm{~nm}$, where the tissue chromophores absorb much less, and can penetrate much greater thicknesses of tissue. Vinogradov and Wilson [19-20] have reported synthesis of a group of oxygen sensitive phosphors (tetrabenzoporphyrins) with absorption maxima near $636 \mathrm{~nm}$ and phosphorescence maxima near $800 \mathrm{~nm}$. The experiments in which a water soluble derivative of Pd-tetrabenzoporphyrin was used to determine the oxygen distribution in the vasculature of tumor bearing mice $[4,11]$ and in the surface and deep areas of the kidney of rats [18] have established that, by using near infra-red phosphors, it is possible to image tissue oxygen distribution through centimeter thicknesses of tissue (such as through the abdomen of an adult mouse). Thus, phosphorescence quenching can now be used both to detect the presence of small regions of hypoxia (such as subcutaneous EMT-6 tumors) present in much larger volumes of normal 


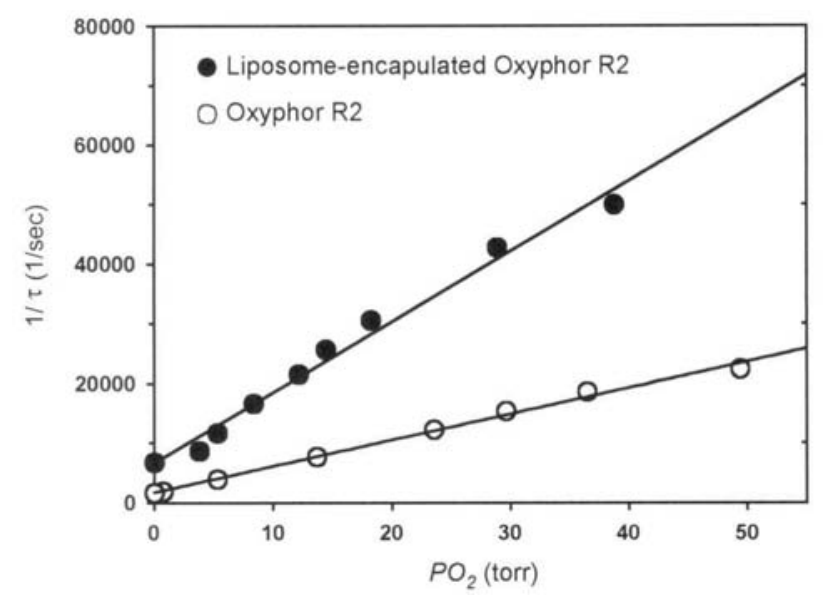

Fig 4. Calibrations of Oxyphor $\mathrm{R} 2$ with $2 \%$ bovine serum albumin with and without liposome encapsulation at $\mathrm{pH} 7.4,38^{\circ} \mathrm{C}$. The $(1 / \tau)$ vs. $\mathrm{PO}_{2}$ delineated Stern-Volmer equation, in which the slope of each plot represented its quenching constant $k_{Q}$. The lifetime at zero oxygen $\tau^{\circ}$ was acquired from the intercept of the plot. The liposome-encapsulated Oxyphor R2 displayed higher quenching constant and shorter lifetime at zero oxygen $\left(k_{\mathrm{O}}=1186 \mathrm{mmHg}^{-1} \mathrm{sec}^{-1} ; \tau^{\circ}=150 \mu \mathrm{sec}\right)$ comparing to Oxyphor $R 2\left(k_{Q}=438 \mathrm{mmHg}^{-1} \mathrm{sec}^{-1}\right.$; $\left.\tau^{\prime}=630 \mu \mathrm{sec}\right)$.

tissue and to quantify the oxygen pressure in such hypoxic tissue volumes. Further design of using liposome to encapsulate the Pd-tetrabenzoporphyrin derivatives that both absorb and phosphoresce at the wavelengths in the near-infrared region should greatly increase the efficiency of using phosphorescence probes for in vivo oxygen measurement, ie., with minimal amount of probes adminstered but to acquire high resolution of quantitative oxygen distribution imaging in vivo. It would take advantages both from liposome in terms of reducing immune response and increasing on-site delivery efficiency, and from the Pdtetrabenzoporphyrin derivatives that posess better tissue penetration depth due to their characteristics of near-infrared phosphorescence.

\section{ACKNOWLEDGEMENTS}

This present work was honored with Research of Excellence in the 2003 Medical Research Competition by the Taiwan Medical Development Foundation. This research was supported by NHRI Intramural Research Grant ME-092-PP-05 from the National Health Research Institutes at Taiwan.

\section{REFERENCE}

1. Vanderkooi $\mathrm{JM}$ and Wilson DF: A new method for measuring oxygen concentration in biology systems. Adv Exptl Med Biol 1986; 200:189-193.

2. Wilson DF, Rumsey WL, and Green, TJ: The oxygen-dependence of mitochondrial oxidative phosphorylation measured by a new optical method for measuring oxygen concentration. J Biol Chem 1988; 263:2712-2718.

3. Wilson DF and Cerniglia GJ: Localization of tumors and evaluation of their state of oxygenation by phosphorescence imaging. Cancer Res 1992; 52:3988-3993.

4. Vinogradov SA, Lo LW, Jenkins WT. and Wilson DF: Noninvasive imaging of the distribution in oxygen in tissue in vivo using near-infrared phosphors. Biophys J 1996; 70:1609-1617.

5. Dunphy I, Vinogradov SA and Wilson DF: Oxyphor R2 and G2: phosphors for measuring oxygen by oxygen-dependent quenching of phosphorescence. Anal Biochem 2002; 310:191198.

6. Vinogradov SA, Maria A and Wilson DF: Frequency domain instrument for measuring phosphorescence lifetime distributions in heterogeneous samples. Rev Sci Instrum 2001; 74:3396-3406.

7. Gewehr P and Delpy DT: An optical oxygen sensor based upon phosphorescence lifetime quenching and employing a polymer immobilised metalloporphyrin probe: I Theory and Instrumentation. Med \& Biol Eng \& Comp 1993; $31: 2-10$.

8. Lo, LW, Huang, SH, Chang, CH, Chen, WY, Tsai, PJ and Yang, CS: A phosphorescence imaging system for monitoring of oxygen distribution in rat liver under ischemia and reperfusion. J Med Biol Eng 2003; 23:19-27.

9. Pawlowski $M$ and Wilson DF: Monitoring of the oxygen pressure in the blood of live animals using the oxygen dependent quenching of phosphorescence. Adv Exptl Med Biol 1992; 316:179-185.

10. Lakowicz JR. Principles of Fluorescence Spectroscopy. Second Edition Academic/Plenum Publishers; 1999.

11. Lo LW, Koch CJ and Wilson DF: Calibration of oxygen-dependent quenching of the phosphorescence of Pd-meso-tetra-(4carboxyphenyl) porphyrin: a phosphor with general application for measuring oxygen concentration in biological systems. Anal Biochem 1996; 236:153160 . 
12. Lo LW, Vinogradov SA, Koch CJ and Wilson DF: A new, water soluble, phosphor for oxygen measurements in vivo. Adv Exptl Med Biol 1997; 428:651-656.

13. Vinogradov SA, Lo LW, and Wilson DF: Dendritic polyglutamic porphyrins: Probing porphyrin protection measured by oxygen-dependent quenching of phosphorescence. Chem Eur J 1999; 5:1338-1347.

14. Yuyama Y, Tsujimoto $M$ and Fujimoto Y: Potential usage of thermosensitive liposomes for sitespecific delivery of cytokines. Cancer Lett 2000; 155:71-77.

15. Li YP, Pei YY, Zhou ZH and Zhang XY: PEGylated polycyanoacrylate nanoparticles as tumor necrosis factor-( carriers. J Control Release 2001; 71:287-296.
16. Bondurant B, Mueller A, OПBrien DF. Photoinitiated destabilization of sterically stabilized liposomes. Biochim Biophys Acta 2001; 1511:113-122.

17. Ho JA A and Wauchope RD. A Strip Liposome Immunoassay for Aflatoxin Bl. Anal Chem 2002; 74:1493-1496.

18. Rumsey WL, Abbott B, Lo LW, Vinogradov SA and Wilson DF: Imaging of oxygen distribution in the surface and deep areas of the kidney. Adv Exptl Med Biol 1997; 411: 591-595.

19. Vinogradov $S A$ and Wilson DF: Metallotetrabenzoporphyrins: New phosphorescent probes for oxygen measurements. J Chem Soc Perkin Trans II 1995; 2:103-111.

20. Vinogradov, SA and Wilson, DF: Extended porphyrins: New IR phosphors for oxygen measurements. Adv Exptl Med Biol 1997; 411:597-604. 